JOURNAL OF

FUNCTION SPACES AND APPLICATIONS

Volume 7, Number 1 (2009), 13-24 (c) 2009, Scientific Horizon

http://www.jfsa.net

\title{
The Jensen functional equation in non-Archimedean normed spaces
}

\author{
Mohammad Sal Moslehian \\ (Communicated by George Isac)
}

2000 Mathematics Subject Classification. Primary 46S10; Secondary 39B52, $39 \mathrm{~B} 82$.

Keywords and phrases. Stability, Jensen functional equation, asymptotic behavior, non-Archimedean normed space.

Abstract. We investigate the Hyers-Ulam-Rassias stability of the Jensen functional equation in non-Archimedean normed spaces and study its asymptotic behavior in two directions: bounded and unbounded Jensen differences. In particular, we show that a mapping $f$ between non-Archimedean spaces with $f(0)=0$ is additive if and only if

$$
\left\|f\left(\frac{x+y}{2}\right)-\frac{f(x)+f(y)}{2}\right\| \rightarrow 0
$$

as $\max \{\|x\|,\|y\|\} \rightarrow \infty$.

\section{Introduction and preliminaries}

The history of the stability theory of functional equations started with a problem concerning group homomorphisms posed by S.M. Ulam [30] in 1940 and its solution given by H.D. Hyers [7] in 1941. Hyers' theorem was generalized by T. Aoki [1] for additive mappings and by Th.M. Rassias [24] for linear mappings by considering an unbounded Cauchy difference. The paper [24] of Th.M. Rassias has provided a lot of influence in the development of what we now call Hyers-Ulam-Rassias stability of functional 
equations. During the last decades many stability problems for various functional equations have been studied by numerous mathematicians. We refer the reader to $[4,8,13,25,26]$ and references therein. The first result on the stability of the classical Jensen equation $f\left(\frac{x+y}{2}\right)=\frac{f(x)+f(y)}{2}$ was given by Z. Kominek [16]. The first author, who investigated the stability problem on a restricted domain was F. Skof [29]. The stability of the Jensen equation and its generalizations were studied by numerous researchers, $\mathrm{cf}$. $[5,12,17,22]$

By a non-Archimedean field we mean a field $K$ equipped with a function (valuation) $|\cdot|$ from $\mathrm{K}$ into $[0, \infty)$ such that $|r|=0$ if and only if $r=0,|r s|=|r||s|$, and $|r+s| \leq \max \{|r|,|s|\}$ for all $r, s \in K$. Clearly $|1|=|-1|=1$ and $|n| \leq 1$ for all $n \in \mathbb{N}$.

Let $X$ be a vector space over a field $\mathbb{K}$ with a non-Archimedean nontrivial valuation $|\cdot|$. A function $\|\cdot\|: X \rightarrow[0, \infty)$ is called a nonArchimedean norm if it satisfies the following conditions:

(i) $\|x\|=0$ if and only if $x=0$;

(ii) $\|r x\|=|r|\|x\| \quad(r \in \mathbb{K}, x \in X)$;

(iii) the strong triangle inequality (ultrametric); namely,

$$
\|x+y\| \leq \max \{\|x\|,\|y\|\} \quad(x, y \in X) .
$$

Then $(X,\|\cdot\|)$ is called a non-Archimedean normed space; cf. [28, 10, 18]. Due to the fact that

$$
\left\|x_{n}-x_{m}\right\| \leq \max \left\{\left\|x_{j+1}-x_{j}\right\|: m \leq j \leq n-1\right\} \quad(n>m)
$$

a sequence $\left\{x_{n}\right\}$ is Cauchy if and only if $\left\{x_{n+1}-x_{n}\right\}$ converges to zero in a non-Archimedean normed space. By a complete non-Archimedean normed space we mean one in which every Cauchy sequence is convergent. Theory of non-Archimedean normed spaces is not trivial, for instance there may not be any unit vector. Although many results in classical normed space theory have a non-Archimedean counterpart, but their proofs are essentially different and require an entirely new kind of intuition, cf. [20, 21, 23].

In 1897, Hensel [6] discovered the $p$-adic numbers as a number theoretical analogue of power series in complex analysis. Fix a prime number $p$. For any nonzero rational number $x$, there exists a unique integer $n_{x} \in \mathbb{Z}$ such that $x=\frac{a}{b} p^{n_{x}}$, where $a$ and $b$ are integers not divisible by $p$. Then $|x|_{p}:=p^{-n_{x}}$ defines a non-Archimedean norm on $\mathbb{Q}$. The completion of $\mathbb{Q}$ with respect to the metric $d(x, y)=|x-y|_{p}$ is denoted by $\mathbb{Q}_{p}$, which is called the $p$-adic number field; cf. $[27,3]$. During the last three decades $p$-adic numbers have gained the interest of physicists for their research in 
particular in problems coming from quantum physics, $p$-adic strings and superstrings; cf. [15].

In [2], the authors investigated stability of approximate additive mappings $f: \mathbb{Q}_{p} \rightarrow \mathbb{R}$. The stability of the Cauchy equation in normed spaces over fields with valuation was studied in [14]. In [20], the stability of Cauchy and quadratic functional equations were investigated in the context of non-Archimedean normed spaces. In this paper, using some ideas from $[9,11,12,19]$ we establish the Hyers-Ulam-Rassias stability of the Jensen functional equation in the setting of non-Archimedean normed spaces and study its asymptotic behavior in two directions: bounded and unbounded Jensen differences.

\section{Stability of the Jensen equation}

In this section, we prove the stability of the Jensen functional equation. Throughout this section we assume that $X$ is a non-Archimedean normed space and $Y$ is a non-Archimedean Banach space over a non-Archimedean field $\mathbb{K}$ with $|3|<1$.

Theorem 2.1. Suppose that $\alpha, \beta \geq 0,0 \leq p<1$ and $f: X \rightarrow Y$ is a mapping satisfying $\|f(0)\| \leq \beta$ and

$$
\left\|f\left(\frac{x+y}{2}\right)-f(x)-f(y)\right\| \leq \alpha \max \left\{\|x\|^{p},\|y\|^{p}\right\} \quad(x, y \in X \backslash\{0\}) .
$$

Then there exists a unique Jensen mapping $T: X \rightarrow Y$ such that

$$
\|f(x)-T(x)\| \leq \max \left\{\frac{\alpha}{|3|^{p}}\|x\|^{p},|2| \beta\right\} \quad(x \in X \backslash\{0\}) .
$$

Proof. Let $x \in X \backslash\{0\}$. Replace $x$ and $y$ by $x$ and $\frac{-x}{3}$ in (2.1), respectively, to get

$$
\begin{aligned}
\left\|2 f\left(\frac{x}{3}\right)-f(x)-f\left(\frac{-x}{3}\right)\right\| & \leq \alpha \max \left\{\|x\|^{p},\left\|\frac{-x}{3}\right\|^{p}\right\} \\
& =\frac{\alpha}{|3|^{p}}\|x\|^{p} \quad\left(\text { by }|3|^{p}<1\right) .
\end{aligned}
$$

Replace $x$ and $y$ by $\frac{x}{3}$ and $\frac{-x}{3}$ in (2.1), respectively, to obtain

$$
\left\|2 f(0)-f\left(\frac{x}{3}\right)-f\left(\frac{-x}{3}\right)\right\| \leq \alpha \max \left\{\left\|\frac{x}{3}\right\|^{p},\left\|\frac{-x}{3}\right\|^{p}\right\}=\frac{\alpha}{|3|^{p}}\|x\|^{p}
$$


16 The Jensen functional equation in non-Archimedean normed spaces

whence

(2.4)

$$
\left\|f\left(\frac{x}{3}\right)+f\left(\frac{-x}{3}\right)\right\| \leq \max \left\{\frac{\alpha}{|3|^{p}}\|x\|^{p},|2|\|f(0)\|\right\} \leq \max \left\{\frac{\alpha}{|3|^{p}}\|x\|^{p},|2| \beta\right\} .
$$

It follows from (2.3) and (2.4) that

$$
\begin{aligned}
\left\|3 f\left(\frac{x}{3}\right)-f(x)\right\| & \leq \max \left\{\left\|2 f(0)-f\left(\frac{x}{3}\right)-f\left(\frac{-x}{3}\right)\right\|,\left\|f\left(\frac{x}{3}\right)+f\left(\frac{-x}{3}\right)\right\|\right\} \\
& \leq \max \left\{\frac{\alpha}{|3|^{p}}\|x\|^{p}, \max \left\{\frac{\alpha}{|3|^{p}}\|x\|^{p},|2| \beta\right\}\right\} \\
& =\max \left\{\frac{\alpha}{|3|^{p}}\|x\|^{p},|2| \beta\right\} \quad(x \in X \backslash\{0\}) .
\end{aligned}
$$

Given $x \in X \backslash\{0\}$, replace $x$ by $\frac{x}{3^{n}}$ in (2.5) and multiple the obtained inequality with $|3|^{n}$ to get

$$
\begin{aligned}
\left\|3^{n+1} f\left(\frac{x}{3^{n+1}}\right)-3^{n} f\left(\frac{x}{3^{n}}\right)\right\| & \leq \max \left\{\frac{\alpha}{|3|^{p}}|3|^{n}\left\|\frac{x}{3^{n}}\right\|^{p},|3|^{n}|2| \beta\right\} \\
& =\max \left\{\left(|3|^{1-p}\right)^{n} \frac{\alpha}{|3|^{p}}\|x\|^{p},|3|^{n}|2| \beta\right\}
\end{aligned}
$$

The right hand side tends to zero as $n \rightarrow \infty$, so the sequence $\left\{3^{n} f\left(\frac{x}{3^{n}}\right)\right\}$ is Cauchy. Since $Y$ is complete, we conclude that $\left\{3^{n} f\left(\frac{x}{3^{n}}\right)\right\}$ is convergent. Set $T(x):=\lim _{n \rightarrow \infty} 3^{n} f\left(\frac{x}{3^{n}}\right)$. Assume that

$$
\left\|3^{n} f\left(\frac{x}{3^{n}}\right)-f(x)\right\| \leq \max \left\{\frac{\alpha}{|3|^{p}}\|x\|^{p},|2| \beta\right\}
$$

for some $n \in \mathbb{N}$. Then

$$
\begin{aligned}
\left\|3^{n+1} f\left(\frac{x}{3^{n+1}}\right)-f(x)\right\| & \leq \max \left\{\left\|3^{n+1} f\left(\frac{x}{3^{n+1}}\right)-3^{n} f\left(\frac{x}{3^{n}}\right)\right\|,\left\|3^{n} f\left(\frac{x}{3^{n}}\right)-f(x)\right\|\right\} \\
& \leq \max \left\{\max \left\{\left(|3|^{1-p}\right)^{n} \frac{\alpha}{|3|^{p}}\|x\|^{p},|3|^{n}|2| \beta\right\},\right. \\
& \left.\max \left\{\frac{\alpha}{|3|^{p}}\|x\|^{p},|2| \beta\right\}\right\} \\
& \max \left\{\frac{\alpha}{|3|^{p}}\|x\|^{p},|2| \beta\right\}
\end{aligned}
$$


Hence (2.6) holds for all positive integer $n$. Letting $n$ approach to infinity in $(2.6)$ we get

$$
\|T(x)-f(x)\| \leq \max \left\{\frac{\alpha}{|3|^{p}}\|x\|^{p},|2| \beta\right\} .
$$

Replacing $x$ and $y$ by $\frac{x}{3^{n}}$ and $\frac{y}{3^{n}}$, respectively, in (2.1) we get

$$
\left\|3^{n} 2 f\left(\frac{x+y}{2.3^{n}}\right)-3^{n} f\left(\frac{x}{3^{n}}\right)-3^{n} f\left(\frac{y}{3^{n}}\right)\right\| \leq \alpha\left(|3|^{1-p}\right)^{n} \max \left\{\|x\|^{p},\|y\|^{p}\right\} .
$$

Taking the limit as $n \rightarrow \infty$ we obtain

$$
2 T\left(\frac{x+y}{2}\right)=T(x)+T(y) \quad(x, y \in X \backslash\{0\}) .
$$

If $T^{\prime}$ is another Jensen mapping satisfying (2.2), then

$$
\begin{aligned}
\left\|T(x)-T^{\prime}(x)\right\| & =\lim _{k \rightarrow \infty}|3|^{k}\left\|T\left(\frac{x}{3^{k}}\right)-T^{\prime}\left(\frac{x}{3^{k}}\right)\right\| \\
& \leq \lim _{k \rightarrow \infty}|3|^{k} \max \left\{\left\|T\left(\frac{x}{3^{k}}\right)-f\left(\frac{x}{3^{k}}\right)\right\|,\left\|f\left(\frac{x}{3^{k}}\right)-T^{\prime}\left(\frac{x}{3^{k}}\right)\right\|\right\} \\
& \leq \lim _{k \rightarrow \infty}|3|^{k} \max \left\{\frac{\alpha}{\left.|3|^{p}\left\|\frac{x}{3^{k}}\right\|^{p},|2| \beta\right\}}\right. \\
& =\lim _{k \rightarrow \infty} \max \left\{\frac{\alpha}{|3|^{p}}\left(|3|^{1-p}\right)^{k}\|x\|^{p},|3|^{k}|2| \beta\right\}=0, \quad x \in X \backslash\{0\} .
\end{aligned}
$$

Therefore $T=T^{\prime}$. This completes the proof of the uniqueness of $T$.

Corollary 2.2. Suppose that $\alpha \geq 0,0 \leq p<1$ and $f: X \rightarrow Y$ is a mapping satisfying $f(0)=0$ and

$$
\left\|f\left(\frac{x+y}{2}\right)-f(x)-f(y)\right\| \leq \alpha \max \left\{\|x\|^{p},\|y\|^{p}\right\} \quad(x, y \in X) .
$$

Then there exists a unique additive mapping $T: X \rightarrow Y$ such that

$$
\|f(x)-T(x)\| \leq \frac{\alpha}{|3|^{p}}\|x\|^{p} \quad(x \in X) .
$$

Proof. It follows from Theorem 2.1 that there is a unique Jensen mapping $T$ satisfying (2.2). Since $f(0)=0$, we have $T(0)=0$. Hence $T$ is clearly additive and satisfies (2.7). 
18 The Jensen functional equation in non-Archimedean normed spaces

\section{Asymptotic aspect of a bounded Jensen difference}

In this section, we deal with the asymptotic behavior of the Jensen functional equation. Throughout this section we assume that $X$ is a nonArchimedean normed space over $\mathbb{K}$ with $\{\|x\|: x \in \mathcal{X}\}=\{|r|: r \in \mathcal{K}\}$ and $Y$ is a non-Archimedean Banach space over a non-Archimedean field $\mathbb{K}$ with $|3|<1$. Utilizing the strategy of Theorem 3 of [12] we get the following result.

Theorem 3.1. Suppose that $\alpha, \beta>0$ and $f: X \rightarrow Y$ is a mapping satisfying $f(0)=0$ and

$$
\left\|f\left(\frac{x+y}{2}\right)-f(x)-f(y)\right\| \leq \alpha
$$

for all $x, y \in X$ with $\max \{\|x\|,\|y\|\} \geq|\beta|$. Then there exists a unique additive mapping $T: X \rightarrow Y$ such that

$$
\|f(x)-T(x)\| \leq \alpha \quad(x \in X) .
$$

Proof. Assume that $\max \{\|x\|,\|y\|\}<|\beta|$. For $x=y=0$ take $z \in X$ to be an element of $X$ with $\|z\|=|\beta|$. Without loss of generality, assume that $\|y\| \leq\|x\|<|\beta|$. Let $\gamma \in K$ with $|\gamma|=\|x\|$. Set $z:=x+\frac{\beta}{3^{n} \gamma} x$ for large enough $n$ such that $x \neq 0$ or $y \neq 0$,

$$
\begin{array}{r}
\max \{\|x-z\|,\|y+z\|\} \geq|\beta| \\
\max \{\|2 z\|,\|x-z\|\} \geq|\beta| \\
\max \{\|y\|,\|2 z\|\} \geq|\beta| \\
\max \{\|y+z\|,\|z\|\} \geq|\beta| \\
\max \{\|x\|,\|z\|\} \geq|\beta| .
\end{array}
$$

It follows from (3.1) and (3.2), we get

$$
\begin{aligned}
\left\|f\left(\frac{x+y}{2}\right)-\frac{f(x)+f(y)}{2}\right\| \leq \max \{ & \left\|f\left(\frac{x+y}{2}\right)-\frac{f(x-z)+f(y+z)}{2}\right\|, \\
& \left\|\frac{f(2 z)+f(x-z)}{2}-f\left(\frac{x+z}{2}\right)\right\|, \\
& \left\|f\left(\frac{y+2 z}{2}\right)-\frac{f(y)+f(2 z)}{2}\right\|, \\
& \left\|\frac{f(y+z)+f(z)}{2}-f\left(\frac{y+2 z}{2}\right)\right\|, \\
& \left.\left\|f\left(\frac{x+z}{2}\right)-\frac{f(x)+f(z)}{2}\right\|\right\} .
\end{aligned}
$$


Thus

$$
\left\|f\left(\frac{x+y}{2}\right)-\frac{f(x)+f(y)}{2}\right\| \leq \alpha
$$

for all $x, y \in X$. Now the result is deduced from Corollary 2.2.

Theorem 3.2. Suppose that $f: X \rightarrow Y$ is a mapping satisfying $f(0)=0$. Then $f$ is additive if and only if

$$
\left\|f\left(\frac{x+y}{2}\right)-\frac{f(x)+f(y)}{2}\right\| \rightarrow 0
$$

as $\max \{\|x\|,\|y\|\} \rightarrow \infty$.

Proof. If $f$ is additive, then (3.3) evidently holds. Conversely, use the limit (3.3) to get for each $n \in \mathbb{N}$ a real number $\beta_{n}>\left|\beta_{n}\right|$ (replace $\beta_{n}$ by a real number of the form $k(n) \beta_{n}$ where $k(n)$ is an integer, if necessary, to get $k(n) \beta_{n}>\left|\beta_{n}\right| \geq\left|k(n) \beta_{n}\right|$, since $\left.|k(n)| \leq 1\right)$ such that

$$
\left\|f\left(\frac{x+y}{2}\right)-\frac{f(x)+f(y)}{2}\right\| \leq 1 / n
$$

for all $x, y \in X$ with

$$
\max \{\|x\|,\|y\|\} \geq \beta_{n}>\left|\beta_{n}\right| .
$$

Next use Theorem 3.1 to conclude a unique additive mapping $T_{n}$ such that

$$
\left\|f(x)-T_{n}(x)\right\| \leq 1 / n
$$

for all $x \in X$. Thus $\left\|f(x)-T_{1}(x)\right\| \leq 1$ and $\left\|f(x)-T_{n}(x)\right\| \leq 1 / n \leq 1$ for each $n$. By the uniqueness of $T_{1}$ we conclude that $T_{n}=T_{1}$ for all $n$. Tending $n$ to infinity in (3.4) we deduce that $f=T_{1}$ is additive.

\section{Asymptotic aspect of an unbounded Jensen difference}

In this section, we deal with the asymptotic behavior of an unbounded Jensen difference. Throughout this section we assume that Let $X$ is a nonArchimedean normed space and $Y$ is a non-Archimedean Banach space over a non-Archimedean field $\mathbb{K}$ with $|2|<1$.

Theorem 4.1. Suppose that $\alpha \geq 0, M>0,0 \leq p<1$ and $f: X \rightarrow Y$ is a mapping satisfying $f(0)=0$ and

$$
\left\|2 f\left(\frac{x+y}{2}\right)-f(x)-f(y)\right\| \leq \alpha \max \left\{\|x\|^{p},\|y\|^{p}\right\}
$$


20 The Jensen functional equation in non-Archimedean normed spaces

for all $x, y \in X$ with $\max \{\|x\|,\|y\|\} \geq M$. Then there exists an additive mapping $T: X \rightarrow Y$ such that

$$
\|f(x)-T(x)\| \leq \alpha\|x\|^{p}
$$

for all $x \in X$ with $\|x\| \geq M$. Furthermore, $T$ is independent of given positive numbers $\alpha$ and $M$.

Proof. Let $\|x\| \geq M$. Put $y=0$ in (4.1) to get

$$
\left\|2 f\left(\frac{x}{2}\right)-f(x)\right\| \leq \alpha\|x\|^{p}
$$

Using (4.3) and the fact that $\left\|x / 2^{n}\right\| \geq M$ for large enough $n$, we can follow the same argument as in the proof of Theorem 2.1 to get a mapping $\tau:\{x \in X:\|x\| \geq M\} \rightarrow Y$ defined by $\lim _{n \rightarrow \infty} 2^{n} f\left(x / 2^{n}\right)$ satisfying

$$
\|\tau(x)-f(x)\| \leq \alpha\|x\|^{p} \quad(\|x\| \geq M) .
$$

and

$$
\tau\left(\frac{x+y}{2}\right)=\frac{1}{2}(\tau(x)+\tau(y))
$$

for all $x, y \in X$ with $\max \{\|x\|,\|y\|\} \geq M$.For each $x \in X$ with $\|x\| \geq M$ we have $\|x / 2\| \geq\|x\| \geq \| M$, whence

$$
\tau(x / 2)=\lim _{n \rightarrow \infty} 2^{n} f\left(x / 2^{n+1}\right)=\frac{1}{2} \lim _{n \rightarrow \infty} 2^{n+1} f\left(x / 2^{n+1}\right)=\frac{1}{2} \tau(x)
$$

Given any $x \in X$ with $0<\|x\|<M$, let $k=k(x)$ denotes the least positive integer such that $\| x / 2^{k}|| \geq M$ and define

$$
T(x)= \begin{cases}0 & x=0 \\ 2^{k} \tau\left(\frac{x}{2^{k}}\right) & \|x\|<M \\ \tau(x) & \|x\| \geq M\end{cases}
$$

Now we show that

$$
T(x)=\lim _{n \rightarrow \infty} 2^{n} f\left(\frac{x}{2^{n}}\right) \quad(x \in X) .
$$

To see this, take any $x \in X$ with $0<\|x\|<M$. Let $k$ be the least positive integer satisfying $\left\|x / 2^{k-1}\right\| \geq M$. Then $k-1$ is the least positive integer satisfying $\left\|\frac{\frac{x}{2}}{2^{k-2}}\right\| \geq M$. Therefore

$$
T(x / 2)=2^{k-1} \tau\left(\frac{\frac{x}{2}}{2^{k-1}}\right)=\frac{1}{2} 2^{k} \tau\left(\frac{x}{2^{k}}\right)=\frac{1}{2} T(x) .
$$


By (4.4) and the definition of $T$, we therefore conclude that

$$
T(x / 2)=\frac{1}{2} T(x)
$$

for all $x \in X$. Let $x \in X \backslash\{0\}$. There is a positive integer $k_{0}$ such that $\left\|2^{-k_{0}} x\right\| \geq M$. We have

$$
\begin{aligned}
T(x)=2^{k_{0}} T\left(2^{-k_{0}} x\right)=2^{k_{0}} \tau\left(2^{-k_{0}} x\right) & =2^{k_{0}} \lim _{n \rightarrow \infty} f\left(\frac{2^{-k_{0}} x}{2^{n}}\right) \\
& =\lim _{n \rightarrow \infty} 2^{n+k_{0}} f\left(\frac{x}{2^{n+k_{0}}}\right)=\lim _{n \rightarrow \infty} 2^{n} f\left(\frac{x}{2^{n}}\right) .
\end{aligned}
$$

Trivially $T(0)=0=\lim _{n \rightarrow \infty} 2^{n} f\left(\frac{0}{2^{n}}\right)$. Hence (4.5) holds for all $x \in X$. If $x=0$ or $y=0$, by taking (4.6) into account, we get $T\left(\frac{x+y}{2}\right)=$ $\frac{1}{2}(T(x)+T(y))$. So we may assume that $x \neq 0$ and $y \neq 0$. Choose $n \in \mathbb{N}$ to be large enough such that

$$
\min \left\{\left\|\frac{x}{2^{n}}\right\|,\left\|\frac{y}{2^{n}}\right\|\right\} \geq M .
$$

Utilizing (4.1) we obtain

$$
\left\|2^{n} f\left(\frac{(x+y) / 2}{2^{n}}\right)-\frac{1}{2} 2^{n} f\left(\frac{x}{2^{n}}\right)-\frac{1}{2} 2^{n} f\left(\frac{y}{2^{n}}\right)\right\| \leq\left(|2|^{1-p}\right)^{n} \max \left\{\|x\|^{p},\|y\|^{p}\right\} .
$$

Letting $n$ approach to infinity we get

$$
T\left(\frac{x+y}{2}\right)=\frac{1}{2}(T(x)+T(y)) .
$$

Hence $T$ is additive.

Suppose that $T^{\prime}$ is another additive mapping satisfying (4.2) with $\alpha$ and $M$ replaced by $\alpha^{\prime}$ and $M^{\prime}$, respectively. For $x \in X$ choose $n \in \mathbb{N}$ large enough so that $\left\|2^{-n} x\right\| \geq \max \left\{M, M^{\prime}\right\}$. Then

$$
\begin{aligned}
\left\|T(x)-T^{\prime}(x)\right\| & =\lim _{k \rightarrow \infty}|2|^{k}\left\|T\left(\frac{x}{2^{k}}\right)-T^{\prime}\left(\frac{x}{2^{k}}\right)\right\| \\
& \leq \lim _{k \rightarrow \infty}|2|^{k} \max \left\{\left\|T\left(\frac{x}{2^{k}}\right)-f\left(\frac{x}{2^{k}}\right)\right\|,\left\|f\left(\frac{x}{2^{k}}\right)-T^{\prime}\left(\frac{x}{2^{k}}\right)\right\|\right\} \\
& \leq \lim _{k \rightarrow \infty}|2|^{k} \max \left\{\alpha\left\|\frac{x}{2^{k}}\right\|^{p}, \alpha^{\prime}\left\|\frac{x}{2^{k}}\right\|^{p}\right\} \\
& =\lim _{k \rightarrow \infty}\left(|2|^{1-p}\right)^{k}\|x\|^{p} \max \left\{\alpha, \alpha^{\prime}\right\} \\
& =0 .
\end{aligned}
$$

Hence $T(x)=T^{\prime}(x)$. 
22 The Jensen functional equation in non-Archimedean normed spaces

Let $f: X \rightarrow Y$ be a mapping. Following [9]

(i) $f$ is called $p$-asymptotic close to an additive mapping $T$ if $\lim _{\|x\| \rightarrow \infty} \frac{\|f(x)-T(x)\|}{\|x\|^{p}}=0$.

(ii) $f$ is said to satisfy $p$-asymptotically the Jensen equation if for each $\alpha>0$ there exists $M>0$ such that

$$
\left\|f\left(\frac{x+y}{2}\right)-f(x)-f(y)\right\| \leq \alpha \max \left\{\|x\|^{p},\|y\|^{p}\right\}
$$

for all $x, y \in X$ with $\max \{\|x\|,\|y\|\} \geq M$.

Applying Theorem 4.1 and the uniqueness of obtained additive mapping we infer the following corollary.

Corollary 4.2. If $0<p<1$ and a mapping $f: X \rightarrow Y$ with $f(0)=0$ satisfies $p$-asymptotically the Jensen equation, then it is $p$-asymptotic close to an additive mapping.

\section{References}

[1] T. Aoki, On the stability of the linear transformation in Banach spaces, J. Math. Soc. Japan, 2 (1950) 64-66.

[2] L.M. Arriola and W.A. Beyer, Stability of the Cauchy functional equation over $p$-adic fields, Real Analysis Exchange, 31 (2005/2006), 125-132.

[3] G. Bachman, Introduction to p-adic Numbers and Valuation Theory, Academic Press, New York-London, 1964.

[4] S. Czerwik, Stability of Functional Equations of Ulam-Hyers-Rassias Type, Hadronic Press, Palm Harbor, Florida, 2003.

[5] V. Faŭziev and P. K. Sahoo, On the stability of Jensen's functional equation on groups, Proc. Indian Acad. Sci. Math. Sci., 117 (2007), 31-48.

[6] K. Hensel, Über eine neue Begründung der Theorie der algebraischen Zahlen, Jahresber. Deutsch. Math. Verein, 6 (1897), 83-88.

[7] D.H. Hyers, On the stability of the linear functional equation, Proc. Nat. Acad. Sci. U.S.A., (1941), 222-224.

[8] D.H. Hyers, G. Isac and Th.M. Rassias, Stability of Functional Equations in Several Variables, Birkhäuser, Basel, 1998.

[9] D.H. Hyers, G. Isac and Th.M. Rassias, On the asymptoticity aspect of Hyers-Ulam stability of mappings, Proc. Amer. Math. Soc., 126 (1998), 425-430. 
[10] G. Isac and G. Marinescu, Analiza pe Corpuri Ultrametrice. (Romanian) [Analysis on Ultrametric Fields], Editura Academiei Republicii Socialiste Romnia, Bucharest, 1976.

[11] G. Isac and Th.M. Rassias, On the Hyers-Ulam stability of $\psi$-additive mappings, J. Approx. Theory, 72 (1993), 131-137.

[12] S.-M. Jung, Hyers-Ulam-Rassias stability of Jensen's equation and its application, Proc. Amer. Math. Soc., 126 (1998), 3137-3143.

[13] S.-M. Jung, Hyers-Ulam-Rassias Stability of Functional Equations in Mathematical Analysis, Hadronic Press lnc., Palm Harbor, Florida, 2001.

[14] Z. Kaiser, On stability of the Cauchy equation in normed spaces over fields with valuation, Publ. Math. Debrecen, 64 (2004), 189-200.

[15] A. Khrennikov, Non-Archimedean Analysis: Quantum Paradoxes, Dynamical Systems and Biological Models, Kluwer Academic Publishers, Dordrecht, 1997.

[16] Z. Kominek, On a local stability of the Jensen functional equation, Demonstratio Math., 22 (1989), 499-507.

[17] Y.-H. Lee and K.-W. Jun, A generalization of the Hyers-Ulam-Rassias stability of Jensen's equation, J. Math. Anal. Appl., 238 (1999), 305315.

[18] A.F. Monna, Analyse Non-Archimédienne. (French) Ergebnisse der Mathematik und ihrer Grenzgebiete, Band 56. Springer-Verlag, BerlinNew York, 1970.

[19] M.S. Moslehian, Asymptotic behavior of the extended Jensen equation, Studia Sci. Math. Hungar., to appear.

[20] M.S. Moslehian and Th.M. Rassias, Stability of functional equations in non-Arhimedian spaces, Appl. Anal. Disc. Math., 1 (2007), 325-334.

[21] M.S. Moslehian and Gh. Sadeghi, A Mazur-Ulam theorem in NonArchimedean normed spaces, Nonlinear Anal., 69 (2008), 3405-3408.

[22] M.S. Moslehian and L. Székelyhidi, Satbility of ternary homomorphisms via generalized Jensen equation, Results in Math., 49 (2006), 289-300.

[23] L. Narici and E. Beckenstein, Strange terrain-non-Archimedean spaces, Amer. Math. Monthly, 88 (1981), 667-676.

[24] Th.M. Rassias, On the stability of the linear mapping in Banach spaces, Proc. Amer. Math. Soc., 72 (1978), 297-300.

[25] Th.M. Rassias, On the stability of functional equations and a problem of Ulam, Acta Appl. Math., 62 (2000), 23-130. 
24 The Jensen functional equation in non-Archimedean normed spaces

[26] Th.M. Rassias, Functional Equations, Inequalities and Applications, Kluwer Academic Publishers, Dordrecht, Boston and London, 2003.

[27] A.M. Robert, A Course in p-adic Analysis, Springer-Verlag, New York, 2000.

[28] P. Schneider, Non-Archimedean Functional Analysis, Springer Monographs in Mathematics, Springer-Verlag, Berlin, 2002.

[29] F. Skof, Sullapprossimazione delle applicazioni localmente $\delta$ - additive, Atti Accad. Sci. Torino, 117 (1983), 377-389.

[30] S.M. Ulam, A collection of mathematical problems Interscience Publ., New York, 1960. Problems in Modern Mathematics, Wiley, New York, 1964.

Department of Pure Mathematics

Ferdowsi University of Mashhad

P. O. Box, 1159, Mashhad 91775

Iran

and

Banach Mathematical Research Group (BMRG)

Mashhad, Iran

Centre of Excellence in Analysis on Algebraic Structures (CEAAS)

Ferdowsi University of Mashhad

Iran

(E-mail : moslehian@ferdowsi.um.ac.ir,moslehian@ams.org)

(Received : October 2007) 


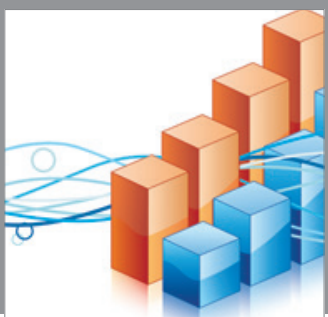

Advances in

Operations Research

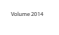

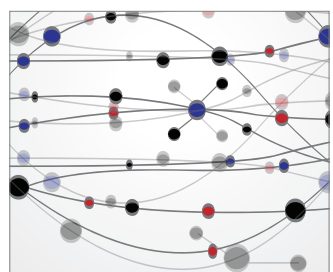

\section{The Scientific} World Journal
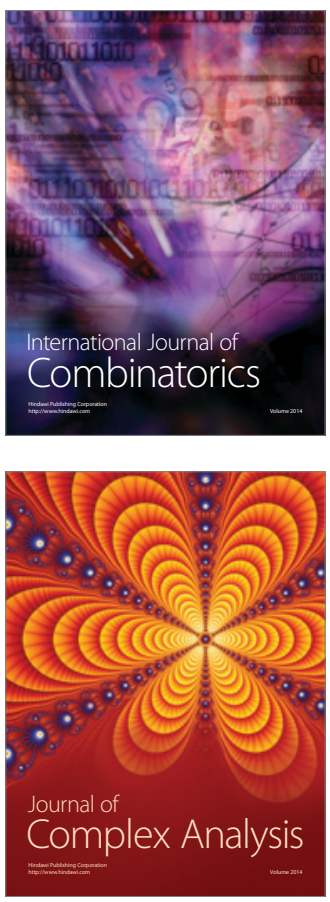

International Journal of

Mathematics and

Mathematical

Sciences
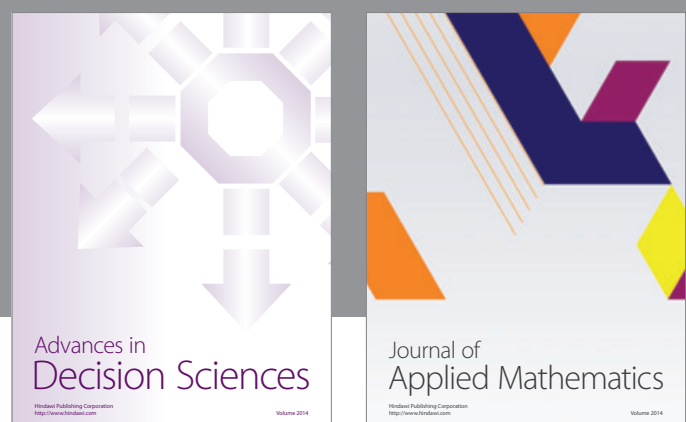

Journal of

Applied Mathematics
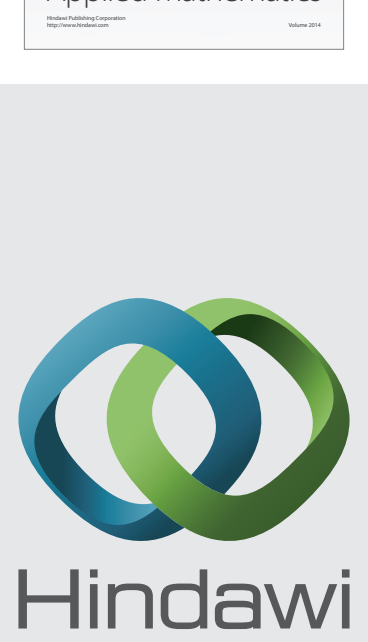

Submit your manuscripts at http://www.hindawi.com
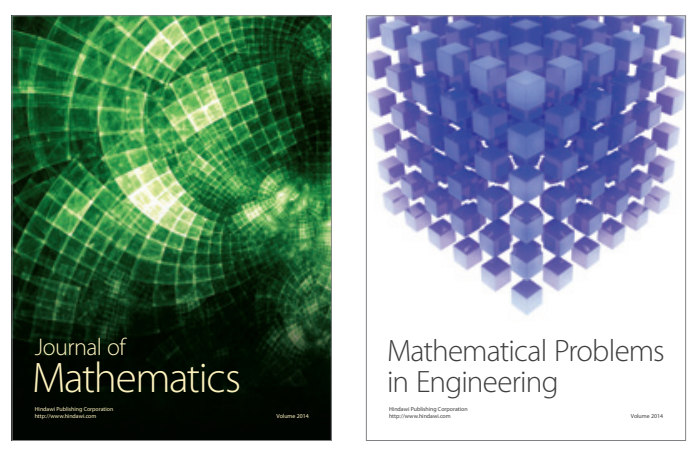

Mathematical Problems in Engineering
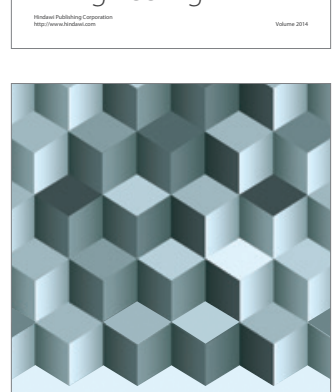

Journal of

Function Spaces
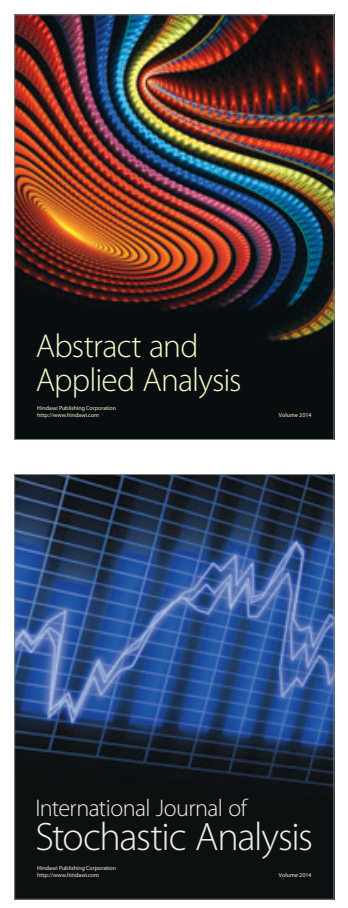

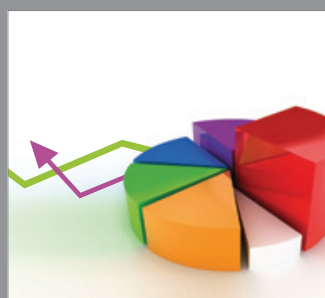

ournal of

Probability and Statistics

Promensencen
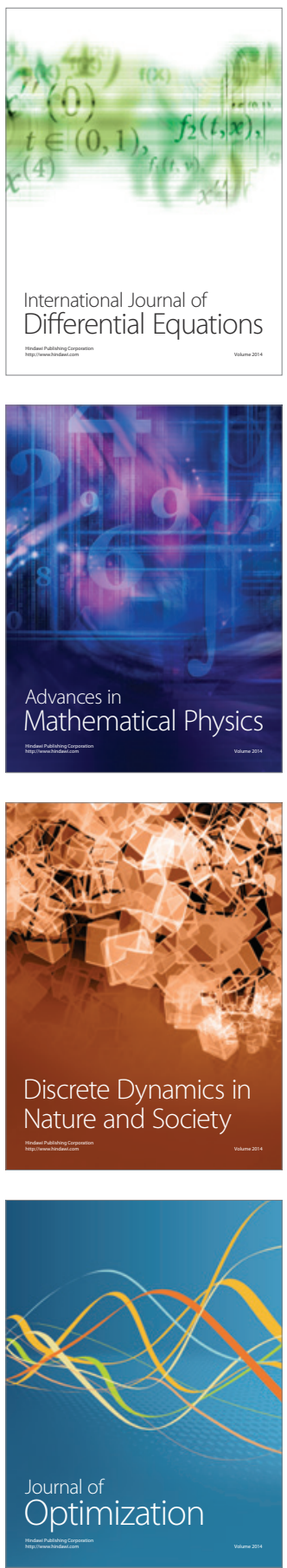